\title{
Chickpea (Cicer arietinum L.) Scenario in India and South Eastern Rajasthan: A Review
}

\author{
Preeti Verma $^{1}$, Rajesh Kumar ${ }^{1}$, R. K. Solanki ${ }^{2}$, Chaman Jadon ${ }^{1}$ and PradeepKumar $^{1 *}$ \\ ${ }^{1}$ Agriculture University, Kota, India \\ ${ }^{2}$ Central Arid Zone Research Institute, Jodhpur, India \\ *Corresponding author
}

\section{A B S T R A C T}

\begin{tabular}{|l|}
\hline Ke y w or d s \\
Chickpea, Pulses, \\
Production, Yield \\
\hline Article Info \\
\hline $\begin{array}{l}\text { Accepted: } \\
\text { 12 December } 2020 \\
\text { Available Online: } \\
\text { 10 January 2021 }\end{array}$ \\
\hline
\end{tabular}

Introduction

Indian agriculture is diverse in nature in terms of agro-ecology and natural resource availability. Self sustainable agriculture systems have evolved with time by integration of pulses as they enrich the soil through symbolic nitrogen fixation from atmosphere. Role of pulses in Indian agriculture, food and nutrition is well known as these crops fit well in crop rotation models; being rich in protein and some of the essential amino acids, acts as a major source of protein to the predominantly vegetarian population of the country. Chickpea is second most important pulse crop worldwide; it is second in area and third in production. The crop meets up to $80 \%$ of the soil's nitrogen needs, so farmers have to apply less nitrogen fertilizer than they do for other non-legume crops. The present paper aims to discuss the scenario of chickpea cultivation in India, Rajasthan state and south eastern Rajasthan (humid south eastern plain zone or zone V) in order to understand the various factors affecting chickpea production and productivity.

Chickpea is classified into desi (chromosome number $2 \mathrm{n}=14,16$ ) and kabuli chickpea (chromosome number $2 \mathrm{n}=16$ ). The desi type however, is more prominent - it accounts for close to $80-85 \%$ and the kabuli type around $15-20 \%$ of the world's total production. India 
produces mostly the desi type chickpeas. It is generally grown under rainfed conditions or in the drier areas of the country as they are best suited for its production but however, gives good returns in irrigated conditions as well. It is best suited to areas having moderate rainfall of 60-90 centimetres per annum, excessive rains after sowing or at flowering and fruiting or hailstorms at ripening cause heavy loss. India is a premier pulse growing country. The domestic demand of chickpea is so huge that inspite of being the largest producer of chickpea, India is also the largest importer of chickpea in the world. The countries which exports chickpea to India are Canada, Australia, Iran, Myanmar, Tanzania, Pakistan, Turkey, France. India also exports chickpea to nations like USA, UK, Saudi Arabia, UAE, Sri Lanka and Malaysia.

The share of chickpea to the total pulses in the country, in terms of area has increased from 29 to 39 percent while in terms of production; it has increased from 42 to 50 percent in last ten years (2004-04 to 2013-14). While there has been a steady increase in area; but fluctuation in production during this decade.The increase in area of the total pulses has been only 9.89 percent while the increase in area of chickpea has been around 48 percent.

The increase in production of total pulses has been around 32 percent while the increase in production of chickpea has been around 74 percent during this period. This quantum increase in area and production of chickpea as compared to total pulses highlights the importance of the crop with respect to pulses as a whole. Inspite of the fluctuation in production, the productivity of chickpea has always been higher than the productivity of all the pulses. Madhya Pradesh contributes highest (39\%), followed by Maharashtra (14\%), Rajasthan (14\%), Andhra Pradesh (10\%), Uttar Pradesh (7\%), Karnataka (6\%) and other remaining states \& UTs of India
$(10 \%)$ to the total chickpea area and production.

Indian Scenario: Since chickpea is the most important pulse crop of India, therefore, a positive association also seems to exist between the production of total pulses and chickpea. Whereas, the production of pulses as well as chickpea has shown an increasing trend during the years 2004-05 to 2006-07, the production of total pulses as well as chickpea has decreased during the years 2011-12 and 2014-15. Excluding the two years i.e., 2007-08 and 2008-09, the production of total pulses and chickpea has been in the same direction during the years 2004-05 to 2006-07 (positive / increase) and from 2009-10 to 2014-15 (either positive/increase or negative/decrease).

The area of chickpea under irrigation in the country has been much higher than the irrigated area under total pulses during the past years. This may account for the higher productivity of chickpea as compared to the total pulses. The highest chickpea area under irrigation is in Madhya Pradesh (53\%) followed by Rajasthan (34\%) which again may be the contributing factor for highest production of chickpea in these two states.

Inspite of being the most important pulse crop, the seed replacement rate of chickpea in India has been observed to be lowest among all the pulses while the seed replacement rate of pigeonpea has always been higher than rest of the pulses.

Seed Replacement Rate (SRR) has gone high due to proactive efforts and various seed related programmes after 2014-15 in most of the pulses. The targeted SRR of $33 \%$ for selfpollinated crops was not only achieved, but also increased with a number of HYVs in the seed chain. The details of SRR is given below (Table-3).

The per capita availability of chickpea per 
annum has increased from $2.9 \mathrm{~kg}$ per year to $5.6 \mathrm{~kg}$ per year during the last decade. It is noteworthy that out of the per capita availability of total pulses, the percent share of chickpea alone had been from 25 to 36 percent, while its share in total foodgrains availability ranges merely between 1.9 to 3.2 percent.The present per capita availability of India is $41.9 \mathrm{~g} /$ day $(15.3 \mathrm{~kg} /$ year $)$, while World Health Organization recommendation is $53 \mathrm{~g} /$ day/capita (19.3 kg/year) (Anonymous 2012), therefore, increasing protein availability through increased pulses production is the way out.

It is remarkable to observe that whenever the total rainfall in a particular year was higher than the average rainfall, the production of pulses and chickpea has also increased significantly. There was around 34 percent increase in production of pulses and chickpea, respectively, when there was an increase of 30 percent in annual rainfall in the year 200304, 24 and 9.89 percent increase in the production of pulses and chickpea, respectively, when there was 24 percent increase in the total rainfall in the year 201011. Similarly in the year 2013-14 also, production of pulses and chickpea increased nearly by 5 and 8 percent when there was 17 percent increase in the total rainfall. It implies that although the maximum area under chickpea cultivation in the country is under rainfed conditions but it seems that pulses as well as chickpea responds well to ample availability of water, may be under critical stages of crop growth and seed setting.

\section{Relationship between actual rainfall and chickpea yield (kg/ha) (Y)}

Simple correlation coefficients (r) were computed to study the relationship between chickpea yield and actual rainfall data (200102 to 2013-14)(Table5; Fig 1). It is clear from the data that seed yield was significantly and positively correlated with rainfall $(\mathrm{R}=0.215)$ The regression coefficients (b) and regression equations were also worked out to quantify the amount of change in seed yield of chickpea for a unit change in rainfall. Result showed that every unit increase in rainfall increased the seed yield of chickpea by 0.351 $\mathrm{kg} / \mathrm{ha}(\mathrm{Y}=276.3+0.351 \mathrm{X})$.

Pigeonpea and chickpea are known as kings of pulses as they together contribute $64 \%$ to total pulses production. Generally, the mojor pest Helicoverpa armigera and disease Fusarium wilt in pigeonpea and chickpea causes 20-40 \% loss to productivity. Therefore, it's essential to intensify research work for developing pest/disease resistant varieties for harvesting high yields.

Chickpea tends to be sensitive to high temperature during the growth and reproductive stages. As the global temperature is rising gradually, therefore, improving heat tolerance in chickpea for enhancing its productivity in warm growing conditions and mitigating impact of climate change becomes a prime concern of the researchers. Many high yielding varieties of chickpea with other desirable traits like disease and insect pest resistance have been developed in the last decade. The seed production programme of these varieties is regularly being taken up as per the annual indent allotted by the Government of India. The major high yielding varieties presently under seed chain procuring highest indent are JAKI 9218, JG 11,Vijay, JG 14, GNG 1581, RVG 203 and GNG 2144 etc. The following varieties have contributed significantly in increasing pulse production in recent times.

JAKI 9218- suitable for cultivation in rainfed conditions, medium large seeded variety

Vijay (Phule G 81-1-1) - suitable for cultivation in rainfed conditions, spreading 
type growth habit, small seeds

RSG 974-suitable for cultivation insuitable for cultivation in rainfed and late sown conditions, resistant to wilt

JG 11-suitable for cultivation in irrigated and rainfed conditions, semi spreading, large pods and seeds

JG 14- heat tolerant variety suitable for late sown conditions

JG 63- suitable for cultivation in rainfed and irrigated conditions, semi spreading with profuse branching

JG 130-suitable for cultivation in rainfed and irrigated conditions, semi spreading, profuse branching, medium tall plant

GNG 1581-suitable for cultivation in normal sown and irrigated, medium plant height, semi erect

RVG 201-suitable for cultivation in irrigated conditions, early maturing, moderately resistant against wilt

RVG 203-suitable for cultivation in irrigated and late sown conditions, moderately resistant against wilt and dry root rot

GNG 2144-suitable for cultivation in irrigated and late sown conditions, tolerance against wilt

GNG 2171-suitable for cultivation in irrigated conditions, tolerance against wilt

As the major area under chickpea is rainfed, hence apart from yield, the desirable characteristics of these leading varieties such as spreading / semi spreading growth habit for suitability for rainfed ecologies, heat tolerance, wilt resistance etc. make them highly indented varieties of the country.

Table.1 Area, production and yield of total pulses and chickpea in India

\begin{tabular}{|l|c|c|c|c|c|c|c|c|}
\hline \multirow{2}{*}{ Year } & \multicolumn{3}{|c|}{ Total Pulses } & \multicolumn{3}{c|}{ Chickpea } & \multicolumn{2}{c|}{ \% share } \\
\cline { 2 - 9 } & $\mathbf{A}$ & $\mathbf{P}$ & $\mathbf{Y}$ & $\mathbf{A}$ & $\mathbf{P}$ & $\mathbf{Y}$ & $\mathbf{A}$ & $\mathbf{P}$ \\
\hline $\mathbf{2 0 0 4}-\mathbf{0 5}$ & 22.76 & 13.13 & 577 & 6.71 & 5.47 & 815 & 29 & $\mathbf{4 2}$ \\
\hline $\mathbf{2 0 0 5}-\mathbf{0 6}$ & 22.39 & 13.39 & 598 & 6.93 & 5.60 & 808 & 31 & $\mathbf{4 2}$ \\
\hline $\mathbf{2 0 0 6}-\mathbf{0 7}$ & 23.76 & 14.20 & 598 & 7.63 & 6.33 & 830 & 32 & $\mathbf{4 5}$ \\
\hline $\mathbf{2 0 0 7}-\mathbf{0 8}$ & 23.81 & 14.76 & 620 & 7.58 & 5.75 & 759 & 32 & $\mathbf{3 9}$ \\
\hline $\mathbf{2 0 0 8}-\mathbf{0 9}$ & 22.99 & 14.57 & 638 & 7.89 & 7.06 & 895 & 34 & $\mathbf{4 8}$ \\
\hline $\mathbf{2 0 0 9}-\mathbf{1 0}$ & 23.35 & 14.66 & 625 & 8.17 & 7.48 & 915 & 35 & $\mathbf{5 1}$ \\
\hline $\mathbf{2 0 1 0}-\mathbf{1 1}$ & 26.28 & 18.24 & 689 & 9.19 & 8.22 & 895 & 35 & $\mathbf{4 5}$ \\
\hline $\mathbf{2 0 1 1}-\mathbf{1 2}$ & 24.46 & 17.09 & 699 & 8.32 & 7.70 & 912 & 34 & $\mathbf{4 5}$ \\
\hline $\mathbf{2 0 1 2}-\mathbf{1 3}$ & 23.47 & 18.34 & 750 & 9.51 & 8.83 & 929 & 41 & $\mathbf{4 8}$ \\
\hline $\mathbf{2 0 1 3}-\mathbf{1 4}$ & 25.26 & 19.25 & 764 & 9.93 & 9.53 & 960 & 39 & $\mathbf{5 0}$ \\
\hline $\mathbf{2 0 1 4}-\mathbf{1 5}$ & 23.55 & 17.15 & 728 & 8.25 & 7.33 & 889 & 35 & $\mathbf{4 3}$ \\
\hline $\mathbf{2 0 1 5}-\mathbf{1 6}$ & 24.91 & 16.35 & 656 & 8.39 & 7.06 & 840 & 34 & $\mathbf{4 3}$ \\
\hline $\mathbf{2 0 1 6}-\mathbf{1 7}$ & 29.44 & 23.13 & 786 & 9.63 & 9.38 & 974 & 33 & $\mathbf{4 0}$ \\
\hline $\mathbf{2 0 1 7}-\mathbf{1 8}$ & $\mathbf{3 1 . 1 1}$ & $\mathbf{2 4 . 5 1}$ & $\mathbf{7 8 8}$ & $\mathbf{1 0 . 7 6}$ & $\mathbf{1 1 . 1 6}$ & $\mathbf{1 0 3 7}$ & $\mathbf{3 4}$ & $\mathbf{4 5}$ \\
\hline
\end{tabular}

Area: m. ha. Production: m. tons Yield: kg/ha

Source: Project Coordinator Report2017-18, AICRP on Chickpea, ICAR-IIPR, Kanpur (U.P.) 
Table.2 All India Yield of Total Pulses and chickpea along with Coverage under Irrigation

\begin{tabular}{|c|c|c|c|c|}
\hline \multirow[t]{2}{*}{ Year } & \multicolumn{2}{|c|}{ Total Pulses } & \multicolumn{2}{|c|}{ Chickpea } \\
\hline & $\begin{array}{c}\text { Yield } \\
\text { (Kg/Hectare) }\end{array}$ & $\begin{array}{c}\text { Area under Irrigation } \\
\qquad(\%)\end{array}$ & $\begin{array}{c}\text { Yield } \\
\text { (Kg/Hectare) }\end{array}$ & $\begin{array}{c}\text { Area under Irrigation } \\
(\%)\end{array}$ \\
\hline 2000-01 & 544 & 12.5 & 744 & 30.9 \\
\hline 2001-02 & 607 & 13.3 & 853 & 30.4 \\
\hline 2002-03 & 543 & 14.4 & 717 & 32.1 \\
\hline 2003-04 & 635 & 13.6 & 811 & 31.0 \\
\hline 2004-05 & 577 & 13.9 & 815 & 31.4 \\
\hline 2005-06 & 598 & 15.0 & 808 & 31.1 \\
\hline 2006-07 & 612 & 15.4 & 845 & 31.7 \\
\hline 2007-08 & 625 & 16.2 & 762 & 34.1 \\
\hline 2008-09 & 659 & 16.0 & 895 & 33.6 \\
\hline 2009-10 & 630 & 16.2 & 915 & 32.2 \\
\hline 2010-11 & 691 & 14.8 & 894 & 29.7 \\
\hline 2011-12 & 699 & 16.1 & 928 & 33.5 \\
\hline 2012-13 & 789 & - & 1036 & - \\
\hline 2013-14* & 764 & - & 967 & - \\
\hline
\end{tabular}

Source: Directorate of Economics and Statistics, Department of Agriculture and Cooperation

Table.3 Crop-wise \& Year-wise SRR (SRR Percent)

\begin{tabular}{|l|c|c|c|}
\hline Crop & $\mathbf{2 0 1 4 - 1 5}$ & $\mathbf{2 0 1 5}-16$ & $\mathbf{2 0 1 6 - 1 7}$ \\
\hline Urd & 30 & 34 & 38 \\
\hline Moong & 24 & 31 & 34 \\
\hline Arhar & 41 & 45 & 48 \\
\hline Pea & 34 & 30 & 30 \\
\hline Gram & 25 & 28 & 32 \\
\hline Lentil & 31 & 27 & 35 \\
\hline
\end{tabular}

Source: Seed Division, Min. of Agri. \& FW (DAC\&FW)

Table.4 Per Capita Net Availability of Food grains (per Annum) in India as on 6 March 2014 (Kg per year)

\begin{tabular}{|c|c|c|c|c|c|}
\hline Year & Gram & Pulses & Food grains & $\begin{array}{c}\text { \% share of gram in total } \\
\text { pulses }\end{array}$ & \% share of gram in total Food grains \\
\hline $\mathbf{2 0 0 1}$ & 2.9 & 10.9 & 151.9 & 26.60 & $\mathbf{1 . 9 0}$ \\
\hline $\mathbf{2 0 0 2}$ & 3.9 & 12.9 & 180.4 & 30.23 & $\mathbf{2 . 1 6}$ \\
\hline $\mathbf{2 0 0 3}$ & 3.1 & 10.6 & 159.7 & 29.24 & $\mathbf{1 . 9 4}$ \\
\hline $\mathbf{2 0 0 4}$ & 4.1 & 13.1 & 168.9 & 31.29 & $\mathbf{2 . 4 2}$ \\
\hline $\mathbf{2 0 0 5}$ & 3.9 & 11.5 & 154.2 & 33.91 & $\mathbf{2 . 4 0}$ \\
\hline $\mathbf{2 0 0 6}$ & 3.9 & 11.8 & 162.5 & 33.05 & $\mathbf{2 . 6 6}$ \\
\hline $\mathbf{2 0 0 7}$ & 4.3 & 12.9 & 161.6 & 33.33 & $\mathbf{2 . 4 4}$ \\
\hline $\mathbf{2 0 0 8}$ & 3.9 & 15.3 & 159.2 & 25.49 & $\mathbf{2 . 8 9}$ \\
\hline $\mathbf{2 0 0 9}$ & 4.7 & 13.5 & 162.1 & 34.81 & $\mathbf{3 . 0 7}$ \\
\hline $\mathbf{2 0 1 0}$ & 4.9 & 12.9 & 159.5 & 37.98 & $\mathbf{3 . 2 0}$ \\
\hline $\mathbf{2 0 1 1}$ & 5.3 & 15.7 & 165.6 & 33.75 & $\mathbf{2 . 9 8}$ \\
\hline $\mathbf{2 0 1 2}$ & 4.9 & 15.2 & 164.2 & 32.23 & $\mathbf{3 . 0 0}$ \\
\hline $\mathbf{2 0 1 3}(\mathbf{P})$ & $\mathbf{5 . 6}$ & $\mathbf{1 5 . 3}$ & $\mathbf{1 8 6 . 4}$ & $\mathbf{3 6 . 6 0}$ & \\
\hline
\end{tabular}

Source: Directorate of Economics and Statistics, Department of Agriculture and Cooperation 
Table.5 All India Rainfall Distribution from 2001-02 to 2013-14

\begin{tabular}{|c|c|c|c|c|c|c|c|}
\hline Year & $\begin{array}{l}\text { P (total } \\
\text { pulses) }\end{array}$ & $\begin{array}{c}\text { P } \\
\text { (chickpea) }\end{array}$ & Actual & Normal & $\begin{array}{l}\text { \% } \\
\text { departure }\end{array}$ & $\begin{array}{l}\text { \% irrigated } \\
\text { area under } \\
\text { total pulses }\end{array}$ & $\begin{array}{l}\text { \% irrigated } \\
\text { area under } \\
\text { chickpea }\end{array}$ \\
\hline $\mathbf{2 0 0 1 - 0 2}$ & 13.37 & 5.47 & 1120.2 & 1196.0 & -6.3 & 13.3 & $\mathbf{3 0 . 4}$ \\
\hline $\mathbf{2 0 0 2 - 0 3}$ & 11.13 & 4.24 & 981.4 & 1205.4 & -18.6 & 14.4 & $\mathbf{3 2 . 1}$ \\
\hline $\mathbf{2 0 0 3 - 0 4}$ & $\mathbf{1 4 . 9 1}$ & $\mathbf{5 . 7 2}$ & 1278.0 & 1196.5 & $\mathbf{6 . 8}$ & 13.6 & $\mathbf{3 1 . 0}$ \\
\hline $\mathbf{2 0 0 4}-\mathbf{0 5}$ & 13.13 & 5.47 & 1085.9 & 1197.3 & -9.3 & 13.9 & $\mathbf{3 1 . 4}$ \\
\hline $\mathbf{2 0 0 5}-\mathbf{0 6}$ & 13.39 & 5.60 & 1185.4 & 1196.8 & -1.0 & 15.0 & $\mathbf{3 1 . 1}$ \\
\hline $\mathbf{2 0 0 6}-\mathbf{0 7}$ & 14.20 & 6.33 & 1133.0 & 1195.5 & -5.2 & 15.4 & $\mathbf{3 1 . 7}$ \\
\hline $\mathbf{2 0 0 7}-\mathbf{0 8}$ & 14.76 & 5.75 & 1180.2 & 1194.8 & -1.2 & 16.2 & $\mathbf{3 4 . 1}$ \\
\hline $\mathbf{2 0 0 8}-\mathbf{0 9}$ & 14.57 & 7.06 & 1075.0 & 1196.4 & -10.1 & 16.0 & $\mathbf{3 3 . 6}$ \\
\hline $\mathbf{2 0 0 9}-\mathbf{1 0}$ & 14.66 & 7.48 & 972.8 & 1195.6 & -18.6 & 16.2 & $\mathbf{3 2 . 2}$ \\
\hline $\mathbf{2 0 1 0}-\mathbf{1 1}$ & $\mathbf{1 8 . 2 4}$ & $\mathbf{8 . 2 2}$ & 1212.3 & 1191.7 & $\mathbf{1 . 7}$ & 14.8 & $\mathbf{2 9 . 7}$ \\
\hline $\mathbf{2 0 1 1}-\mathbf{1 2}$ & 17.09 & 7.70 & 1094.7 & 1186.9 & -7.8 & 16.1 & $\mathbf{3 3 . 5}$ \\
\hline $\mathbf{2 0 1 2}-\mathbf{1 3}$ & 18.34 & 8.83 & 1073.4 & 1186.3 & -9.5 & - & - \\
\hline $\mathbf{2 0 1 3}-\mathbf{1 4}$ & $\mathbf{1 9 . 2 5}$ & $\mathbf{9 . 5 3}$ & 1262.4 & 1186.3 & $\mathbf{6 . 4}$ & - & - \\
\hline $\mathbf{2 0 1 4}-\mathbf{1 5}$ & $\mathbf{1 7 . 3 8}$ & $\mathbf{7 . 1 7}$ & & & & & \\
\hline $\mathbf{S}$ & & & & & - \\
\hline
\end{tabular}

Source: Directorate of Economics and Statistics, Department of Agriculture and Cooperation

Table.6 Chickpea Breeder seed indent of high yielding varieties during last ten years

\begin{tabular}{|c|c|c|c|c|}
\hline Year & $\begin{array}{l}\text { Total indent of chickpea } \\
\text { (q) }\end{array}$ & Variety & $\begin{array}{l}\text { DAC Indent } \\
\text { (q) }\end{array}$ & $\begin{array}{l}\% \text { share of the } \\
\text { variety }\end{array}$ \\
\hline \multirow[t]{9}{*}{ 2010-11 } & 9888.84 & JG 11 & 1123.50 & 11.36 \\
\hline & & A 1(Annegiri 1) & 760.70 & 7.69 \\
\hline & & Vijay & 526.60 & 5.32 \\
\hline & & RSG 888 & 450.00 & 5.01 \\
\hline & & JG 130 & 443.00 & 4.47 \\
\hline & & JG 63 & 408.00 & 4.12 \\
\hline & & JAKI 9218 & 420.00 & 4.24 \\
\hline & & JG 322 & 400.00 & 4.04 \\
\hline & & GNG 1581 & 339.70 & 3.43 \\
\hline \multirow[t]{8}{*}{ 2011-12 } & 9367.94 & JG 11 & 937.80 & 10.01 \\
\hline & & Vijay (Phule G 81-1-1) & 584.70 & 6.24 \\
\hline & & JAKI 9218 & 572.00 & 6.10 \\
\hline & & JG 63 & 470.00 & 5.01 \\
\hline & & GNG 1581 & 455.70 & 4.86 \\
\hline & & JG 130 & 425.00 & 4.53 \\
\hline & & Digvijay & 359.05 & 3.83 \\
\hline & & JG 14 & 302.00 & 3.22 \\
\hline \multirow[t]{6}{*}{ 2012-13 } & 9367.94 & JG 11 & 937.80 & 10.01 \\
\hline & & Vijay (Phule G 81-1-1) & 584.70 & 6.24 \\
\hline & & JG 63 & 470.00 & 5.01 \\
\hline & & GNG 1581 & 455.70 & 4.86 \\
\hline & & JG 322 & 450.00 & 4.80 \\
\hline & & JG 130 & 425.00 & 4.53 \\
\hline
\end{tabular}




\begin{tabular}{|c|c|c|c|c|}
\hline \multirow[t]{7}{*}{ 2013-14 } & 9433.23 & JG 6 & 1087.00 & 11.52 \\
\hline & & JAKI 9218 & 910.00 & 9.64 \\
\hline & & GNG 1581 & 799.40 & 8.47 \\
\hline & & JG 11 & 587.00 & 6.22 \\
\hline & & JG 63 & 509.00 & 5.39 \\
\hline & & JG 130 & 472.00 & 5.00 \\
\hline & & KPG 59 & 391.25 & 4.14 \\
\hline \multirow[t]{7}{*}{ 2014-15 } & 6741.6 & JAKI 9218 & 964.65 & 14.30 \\
\hline & & GNG 1581 & 763.60 & 11.32 \\
\hline & & JG 11 & 565.30 & 8.38 \\
\hline & & JG 315 & 407.00 & 6.03 \\
\hline & & JG 322 & 347.00 & 5.14 \\
\hline & & JG 14 & 334.00 & 4.95 \\
\hline & & JG 130 & 308.00 & 4.56 \\
\hline \multirow{7}{*}{ 2015-16 } & 7184.34 & GNG 1581 & 933.70 & 12.99 \\
\hline & & JAKI 9218 & 916.90 & 12.76 \\
\hline & & JG 11 & 525.60 & 7.31 \\
\hline & & RSG 974 & 491.00 & 6.83 \\
\hline & & JG 14 & 411.60 & 5.72 \\
\hline & & JG 63 & 374.20 & 5.20 \\
\hline & & JG 130 & 344.30 & 4.79 \\
\hline \multirow[t]{7}{*}{ 2016-17 } & 10119.41 & JAKI 9218 & 1875.00 & 18.52 \\
\hline & & JG 11 & 1199.70 & 11.85 \\
\hline & & RSG 974 & 580.00 & 5.73 \\
\hline & & Digvijay & 576.70 & 5.01 \\
\hline & & JG 63 & 417.00 & 4.12 \\
\hline & & JG 14 & 396.80 & 3.92 \\
\hline & & GNG 1581 & 388.00 & 3.83 \\
\hline \multirow[t]{9}{*}{ 2017-18 } & 10226.36 & JAKI-9218 & 1593.10 & 15.57 \\
\hline & & JG 11 & 1073.25 & 10.49 \\
\hline & & JG 14 & 418.80 & 4.09 \\
\hline & & RSG 974 & 307.00 & 3.00 \\
\hline & & CSJ 515 & 316.80 & 3.09 \\
\hline & & GNG 1958 & 458.60 & 4.48 \\
\hline & & Raj Vijay Gram 203 & 406.30 & 3.97 \\
\hline & & GNG 2171 & 520.60 & 5.09 \\
\hline & & NBeG 119 & 400.00 & 3.91 \\
\hline \multirow[t]{5}{*}{ 2018-19 } & 9790.16 & Raj Vijay Gram 202 & 1005.00 & 10.26 \\
\hline & & Raj Vijay Gram 203 & 1028.00 & 10.50 \\
\hline & & JG 11 & 984.70 & 10.05 \\
\hline & & JAKI-9218 & 831.70 & 8.49 \\
\hline & & Raj Vijay Gram 201 & 720.00 & 7.35 \\
\hline \multirow[t]{7}{*}{ 2019-20 } & 10678.64 & Raj Vijay Gram 202 & 1001.6 & 9.37 \\
\hline & & Raj Vijay Gram 203 & 927.00 & 8.68 \\
\hline & & GNG 2144 & 769.20 & 7.20 \\
\hline & & JAKI-9218 & 621.30 & 5.81 \\
\hline & & JG 11 & 620.00 & 5.80 \\
\hline & & Raj Vijay Gram 201 & 605.00 & 5.66 \\
\hline & & GNG 2171 & 553.00 & 5.17 \\
\hline
\end{tabular}


Table.7 Area, production and yield of chickpea in India and Rajasthan

\begin{tabular}{|l|c|c|c|c|c|c|c|c|}
\hline \multirow{2}{*}{ Year } & \multicolumn{3}{|c|}{ Total } & \multicolumn{3}{c|}{ State } & \multicolumn{2}{c|}{ \% Share of state } \\
\cline { 2 - 10 } & $\mathbf{A}$ & $\mathbf{P}$ & $\mathbf{Y}$ & $\mathbf{A}$ & $\mathbf{P}$ & $\mathbf{Y}$ & $\mathbf{A}$ & $\mathbf{P}$ \\
\hline $\mathbf{2 0 0 4} \mathbf{- 0 5}$ & 6.71 & 5.47 & 815 & 1035.20 & 773.00 & 747 & 15 & $\mathbf{1 4}$ \\
\hline $\mathbf{2 0 0 5 - 0 6}$ & 6.93 & 5.60 & 808 & 1081.90 & 478.90 & 443 & 16 & $\mathbf{9}$ \\
\hline $\mathbf{2 0 0 6 - 0 7}$ & 7.63 & 6.33 & 830 & 1010.80 & 872.60 & 863 & 13 & $\mathbf{1 4}$ \\
\hline $\mathbf{2 0 0 7 - 0 8}$ & 7.58 & 5.75 & 759 & 1231.30 & 574.20 & 466 & 16 & $\mathbf{1 0}$ \\
\hline $\mathbf{2 0 0 8 - 0 9}$ & 7.89 & 7.06 & 895 & 1260.00 & 980.00 & 779 & 16 & $\mathbf{1 4}$ \\
\hline $\mathbf{2 0 0 9 - 1 0}$ & 8.17 & 7.48 & 915 & 880.00 & 530.00 & 604 & 11 & $\mathbf{7}$ \\
\hline $\mathbf{2 0 1 0 - 1 1}$ & 9.19 & 8.22 & 895 & 1780.00 & 1600.00 & 899 & 19 & $\mathbf{1 9}$ \\
\hline $\mathbf{2 0 1 1 - 1 2}$ & 8.32 & 7.70 & 912 & 1430.00 & 990.00 & 691 & 17 & $\mathbf{1 3}$ \\
\hline $\mathbf{2 0 1 2}-13$ & 9.51 & 8.83 & 929 & 1252.00 & 1265.20 & 1010 & 13 & $\mathbf{1 4}$ \\
\hline $\mathbf{2 0 1 3 - 1 4}$ & 9.93 & 9.53 & 960 & 1923.00 & 1640.40 & 853 & 19 & $\mathbf{1 7}$ \\
\hline $\mathbf{2 0 1 4 - 1 5}$ & 8.25 & 7.33 & 889 & 1256.30 & 911.10 & 725 & 15 & $\mathbf{1 2}$ \\
\hline $\mathbf{2 0 1 5}-16$ & 8.39 & 7.06 & 840 & 941.95 & 840.34 & 892 & 11 & $\mathbf{1 2}$ \\
\hline $\mathbf{2 0 1 6 - 1 7}$ & 9.63 & 9.38 & 974 & 1547.91 & 1409.39 & 911 & 16 & $\mathbf{1 5}$ \\
\hline $\mathbf{2 0 1 7}-18$ & $\mathbf{1 0 . 7 6}$ & $\mathbf{1 1 . 1 6}$ & $\mathbf{1 0 3 7}$ & $\mathbf{1 5 7 2 . 4 8}$ & $\mathbf{1 6 7 0 . 2 6}$ & $\mathbf{1 0 6 2}$ & $\mathbf{1 5}$ & $\mathbf{1 5}$ \\
\hline
\end{tabular}

Area: thousand ha. Production: thousand tons Yield: $\mathrm{kg} / \mathrm{ha}$

Source: Project Coordinator's Report, AICRP on Chickpea, ICAR-IIPR, Kanpur (U.P.)

Table.8 Area, production, productivity of chickpea in zone V and Rajasthan

\begin{tabular}{|c|c|c|c|c|c|c|c|c|}
\hline \multirow[t]{2}{*}{ Year } & \multicolumn{3}{|c|}{ Area (ha) } & \multicolumn{3}{|c|}{ Production (t) } & \multicolumn{2}{|c|}{$\begin{array}{l}\text { Productivity } \\
\text { (kg/ha) }\end{array}$} \\
\hline & State & $\begin{array}{c}\text { Zone } \\
\text { V }\end{array}$ & $\begin{array}{c}\text { \%share of } \\
\text { zone V }\end{array}$ & State & Zone V & $\begin{array}{c}\text { \%share of } \\
\text { zone V }\end{array}$ & State & Zone V \\
\hline 2000-01 & 692634 & 57766 & 8.34 & 406576 & 46270 & 11.38 & 587 & 801 \\
\hline 2001-02 & 969626 & 78734 & 8.12 & 735946 & 74246 & 10.08 & 759 & 943 \\
\hline 2002-03 & 449680 & 28105 & 6.25 & 340407 & 32236 & 9.46 & 757 & 1147 \\
\hline 2003-04 & 1117506 & 43359 & 3.88 & 707381 & 39239 & 5.54 & 633 & 905 \\
\hline 2004-05 & 1036792 & 27579 & 2.66 & 773000 & 23607 & 3.05 & 746 & 856 \\
\hline $2005-06$ & 1081932 & 16554 & 1.53 & 478900 & 14600 & 3.04 & 443 & 882 \\
\hline 2006-07 & 1010769 & 29717 & 2.94 & 872600 & 34798 & 3.98 & 863 & 1171 \\
\hline 2007-08 & 1231282 & 50113 & 4.07 & 574200 & 47306 & 8.23 & 466 & 944 \\
\hline 2008-09 & 1259474 & 43830 & 3.48 & 980000 & 46109 & 4.70 & 779 & 1052 \\
\hline 2009-10 & 884358 & 63939 & 7.23 & 530000 & 58440 & 11.02 & 605 & 914 \\
\hline 2010-11 & 1783281 & 61102 & 3.42 & 1600718 & 58449 & 3.65 & 898 & 957 \\
\hline 2011-12 & 1433928 & 30332 & 2.11 & 989986 & 35990 & 3.63 & 690 & 1187 \\
\hline 2012-13 & 1252936 & 35731 & 2.85 & 1277348 & 44748 & 3.50 & 1019 & 1252 \\
\hline 2013-14 & 1923501 & 40320 & 2.09 & 16407464 & 379814 & 2.31 & 853 & 942 \\
\hline 2014-15 & 1256323 & 22146 & 1.76 & 911085 & 22727 & 2.49 & 725 & 1026 \\
\hline 2015-16 & 941950 & 22659 & 2.40 & 840341 & 27855 & 3.31 & 892 & 1229 \\
\hline 2016-17 & 1547908 & 46614 & 3.01 & 1409387 & 71823 & 5.09 & 911 & 1541 \\
\hline 2017-18 & 1572487 & - & - & 1670265 & - & - & 1062 & - \\
\hline
\end{tabular}

Source: Vital Agricultural Statistics, Government of Rajasthan 2017-18 
Table.9 Breeder seed indent of chickpea in Rajasthan during last five years

\begin{tabular}{|c|c|c|c|c|c|}
\hline Year & Centre & $\begin{array}{c}\text { DAC } \\
\text { Indent }\end{array}$ & $\begin{array}{c}\text { Allotment } \\
\text { BSP-I }\end{array}$ & Production & Surplus/deficit \\
\hline \multirow[t]{4}{*}{ 2014-15 } & SKRAU, Bikaner & 344.00 & 378.50 & 514.30 & 170.30 \\
\hline & AU, Kota & 241.20 & 263.00 & 317.00 & 75.80 \\
\hline & SKNAU, Jobner & 415.60 & 455.90 & 406.01 & -9.59 \\
\hline & MPUAT, Udaipur & 18.00 & 20.00 & 51.00 & 33.00 \\
\hline \multicolumn{2}{|c|}{ State Total } & 1018.80 & 1117.40 & 1288.31 & 266.51 \\
\hline \multicolumn{2}{|c|}{$\%$ share of AU, Kota } & 23.67 & 23.53 & 24.60 & \\
\hline \multirow[t]{4}{*}{$2015-16$} & SKRAU, Bikaner & 367.5 & 404 & 615 & 247.5 \\
\hline & AU, Kota & 395.2 & 458 & 603 & 207.8 \\
\hline & SKNAU, Jobner & 712.6 & 787 & 679 & -33.6 \\
\hline & MPUAT, Udaipur & 95.7 & 106 & 85 & -10.7 \\
\hline \multicolumn{2}{|c|}{ State Total } & 1571 & & & \\
\hline \multicolumn{2}{|c|}{$\%$ share of AU, Kota } & 25.15 & & & \\
\hline \multirow[t]{4}{*}{ 2016-17 } & SKRAU, Bikaner & 322 & 408 & 454 & 132 \\
\hline & AU, Kota & 407.3 & 510 & 1085 & 677.7 \\
\hline & SKNAU, Jobner & 392.5 & 491 & 335.3 & -57.2 \\
\hline & MPUAT, Udaipur & 122 & 152 & 88 & -34.0 \\
\hline \multicolumn{2}{|c|}{ State Total } & 1243.8 & 1561 & 1962.3 & \\
\hline \multicolumn{2}{|c|}{$\%$ share of AU, Kota } & 32.74 & 32.67 & 55.29 & \\
\hline \multirow[t]{4}{*}{$2017-18$} & SKRAU, Bikaner & 631.6 & 731.39 & 633 & 1.4 \\
\hline & AU, Kota & 344.8 & 400 & 646.8 & 302 \\
\hline & SKNAU, Jobner & 309 & 355.32 & 277.8 & -31.2 \\
\hline & MPUAT, Udaipur & 25 & 28.75 & 84.54 & 59.54 \\
\hline \multicolumn{2}{|c|}{ State Total } & 1310.4 & 1515.46 & 1642.14 & 331.74 \\
\hline \multicolumn{2}{|c|}{$\%$ share of AU, Kota } & 26.31 & 26.39 & 39.38 & \\
\hline \multirow[t]{4}{*}{ 2018-19 } & SKRAU, Bikaner & 759.3 & 860 & 869 & 109.7 \\
\hline & AU, Kota & 659.5 & 815 & 876.84 & 217.34 \\
\hline & SKNAU, Jobner & 176 & 202.4 & 259 & 83 \\
\hline & MPUAT, Udaipur & 20 & 23 & 54.37 & 34.37 \\
\hline \multicolumn{2}{|c|}{ State Total } & 1614.8 & 1900.4 & 2059.21 & 444.41 \\
\hline \multicolumn{2}{|c|}{$\%$ share of AU, Kota } & 40.84 & 42.88 & 42.58 & \\
\hline
\end{tabular}

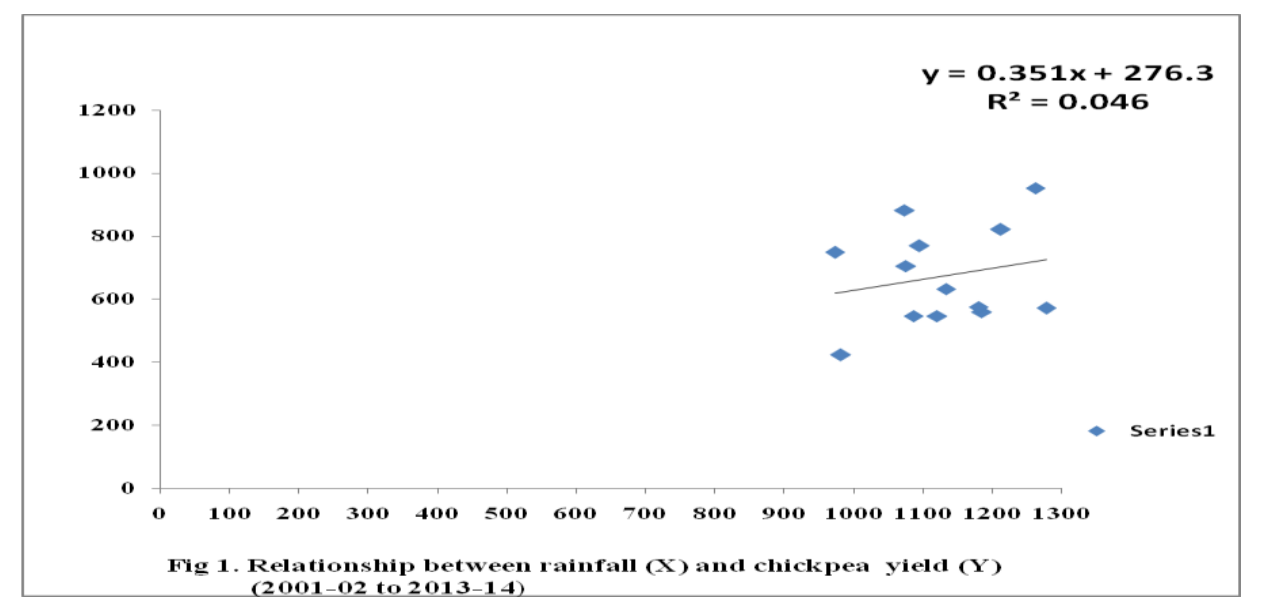




\section{Scenario of chickpea cultivation in South Eastern Rajasthan (zone V):}

Rajasthan is one of the major chickpea producing state of the country. Few facts of state chickpea production, productivity and cultivation regions are as under:

The share of Rajasthan state to the country's total area has increased from 15 to 19 percent while in production; it has increased from 14 to 17 percent in last ten years.

The area of chickpea in Rajasthan has increased from 692634 ha in 2000-01 to 1547908 ha in 2016-17i.e. there has been an increase of 81 percent area in last 15 years.

In contrast to the 81 percent increase in the area of chickpea, the production of the crop has increased to 124 percent during the same period as it has increased from 406576 tons in 2000-01 to 1409387 tons in 2016-17. The increase in production is higher as compared to the increase in area which can be attributed to the cultivation of high yielding, disease and insect-pest resistant varieties along with better crop production techniques.

Despite of such a huge increase in area as well as production, the productivity of the crop in the state has shown an increase of a mere 23.5 percent in the same period. The reasons for this slow growth in productivity need to be examined thoroughly so as to remove the impediments in raising the productivity. The probable reasons might be post harvest losses due to storage pests andlosses during grading and processing.

The area of chickpea in zone V (Humid south eastern plain zone) of the state is very less as compared to the total area of the crop in the state. The area has generally always shown a decreasing trend in the last 15 years. It has dropped down from
8.34 percent to 3.01 percent i.e. around 64 percent decrease in the period of last15 years. The probable reason may be cultivation of wheat, mustard and other remunerative crops instead of chickpea in areas with assured irrigation facilities, as chickpea is generally cultivated as rainfed crop.

The production of chickpea in the zone has increased from 46270 tons in $2000-01$ to 71823 tons in 2016-17 showing a gain of 55 percent. The remarkable thing is that inspite of the huge decrease in area; there has been an increase in production. It can again be attributed to the cultivation of high yielding, disease and insect-pest resistant varieties, better crop production techniques along with higher fertility status, owing to presence of clay loam soil in the zone.

Inspite of a very insignificant area, the productivity of chickpea in zone $\mathrm{V}$ (south eastern humid plain zone) has always remained higher than the productivity of chickpea in Rajasthan state.

The productivity in zone $\mathrm{V}$ has increased from $801 \mathrm{~kg} / \mathrm{ha}$ in $2000-01$ to $1541 \mathrm{~kg} / \mathrm{ha}$ in 2016-17 i.e. there has been an increase of 92 percent in the productivity of zone $\mathrm{V}$ in last 15 years while the productivity of the state has shown an increase of 23.5 percent in the last 15 years.

The major high yielding varieties of chickpea under cultivation in the state are GNG 2171, GNG 2144, GNG 1958, GNG 1581 and CSJ 515 while GNG 469, Pratap Chana-1,GNG 1958 and CSJ 515 are the major varieties recommended for cultivation in the zone.

In conclusion the view of the various chickpea facts and figures related to area, production, productivity, rainfall pattern, irrigation availability, prominent varieties under cultivation, it can be concluded that chickpea production and productivity can be 
raised through cumulative effect of adoption of suitable high yielding, abiotic and biotic stress resistant varieties, better crop production and protection practices in order to reduce import, saving our currency and achieve self-sufficiency.

\section{References}

Agricultural Statistics at a glance 2014 . Singh, R.P. Status paper on pulses. Vital Agricultural Statistics. Annual Reports, AICRP on Chickpea

\section{How to cite this article:}

Preeti Verma, Rajesh Kumar, R. K. Solanki, Chaman Jadon and Pradeep Kumar. 2021. Chickpea (Cicer arietinum L.) Scenario in India and South Eastern Rajasthan: A Review. Int.J.Curr.Microbiol.App.Sci. 10(01): 1057-1067. doi: https://doi.org/10.20546/ijcmas.2021.1001.128 